\title{
Invariants for a Class of Nongeneric Three-qubit States
}

\author{
Bao-Zhi Sun ${ }^{a}$, Shao-Ming Fei ${ }^{a, b}$ \\ ${ }^{a}$ Department of Mathematics, Capital Normal University, Beijing 100037 \\ ${ }^{b}$ Max-Planck-Institute for Mathematics in the Sciences, 04103 Leipzig
}

\begin{abstract}
We investigate the equivalence of quantum states under local unitary transformations. A complete set of invariants under local unitary transformations is presented for a class of non-generic three-qubit mixed states. It is shown that two such states in this class are locally equivalent if and only if all these invariants have equal values for them.

PACS numbers: 03.67.-a, 02.20.Hj, 03.65.-w
\end{abstract}

Key words: Invariant, quantum state, local unitary transformation

Quantum entangled states are playing very important roles in quantum information processing and quantum computation [1]. As the properties of entanglement of a multipartite quantum system remain invariant under local unitary transformations on the subsystems, the entanglement can be characterized by all the invariants of the local unitary transformations. The trace norms of realigned or partial transposed density matrices in entanglement measure and separability criteria are some of these invariants [2]. Invariants are also relevant in the discussions of Bell inequalities [3, 4] and teleportation, etc. 5]. A complete set of invariants gives rise to the classification of the quantum states under local unitary transformations. Two quantum states are locally equivalent if and only if all these invariants have equal values for these states. For bipartite mixed states, a generally non-operational method has been presented to compute all the invariants of local unitary transformations in [6. 7]. In [8], the invariants for general two-qubit systems are studied and a complete set of 18 polynomial invariants is presented. In [9, 10, complete sets of invariants for some classes 
of density matrices have been presented. The invariants for tripartite pure states have been also studied [11].

In [12, the invariants for three qubits states have been discussed. A complete set of invariants for generic mixed states are presented. In this paper we investigate the invariants for non-generic three-qubit states, and present some new invariants for a class of such nongeneric three-qubit states. These invariants plus the invariants given in [12] make the complete set of invariants for these states. Any two of these density matrices are locally equivalent if and only if all these invariants have equal values for these density matrices.

The density matrices of three qubits may be written as :

$$
\begin{aligned}
\rho= & \frac{1}{8}\left(I_{2} \otimes I_{2} \otimes I_{2}+\alpha_{i} \sigma_{i} \otimes I_{2} \otimes I_{2}+\beta_{i} I_{2} \otimes \sigma_{i} \otimes I_{2}+\gamma_{i} I_{2} \otimes I_{2} \otimes \sigma_{i}\right. \\
& \left.+R_{i j} \sigma_{i} \otimes \sigma_{j} \otimes I_{2}+S_{i j} \sigma_{i} \otimes I_{2} \otimes \sigma_{j}+T_{i j} I_{2} \otimes \sigma_{i} \otimes \sigma_{j}+Q_{i j k} \sigma_{i} \otimes \sigma_{j} \otimes \sigma_{k}\right),
\end{aligned}
$$

where $\sigma_{i}$ are pauli matrices, the repeated indices are assumed to be summed over from 1 to 3 . If one considers the qubits as spin- $1 / 2$ particles, the three dimensional vectors $\alpha=\left(\alpha_{1}, \alpha_{2}, \alpha_{3}\right), \beta=\left(\beta_{1}, \beta_{2}, \beta_{3}\right), \gamma=\left(\gamma_{1}, \gamma_{2}, \gamma_{3}\right)$ are their average spins in the state $\rho$, while $R_{i j}, S_{i j}, T_{i j}$ are spin-spin correlators respectively, and $Q_{i j k}$ are three spin correlators. Any local unitary transformations on $\rho$ can be represented by three corresponding $3 \times 3$ orthogonal real matrices of "spin rotation", $L, M, N \in S O(3, \mathbb{R})$. The operation, $L \otimes$ $M \otimes N$, transforms $\rho$ according to the following rules: $\alpha \rightarrow L \alpha, \beta \rightarrow M \beta, \gamma \rightarrow N \gamma, R \rightarrow$ $L R M^{T}, S \rightarrow L S N^{T}, T \rightarrow M T N^{T}, Q^{(1)} \rightarrow L Q^{(1)} M^{T} \otimes N^{T}$, where the last relation can be also written as: $Q^{(2)} \rightarrow M Q^{(2)} L^{T} \otimes N^{T}$ or $Q^{(3)} \rightarrow N Q^{(3)} L^{T} \otimes M^{T}$, with

$$
\begin{aligned}
& Q^{(1)}=\left(\begin{array}{lllllllll}
Q_{111} & Q_{112} & Q_{113} & Q_{121} & Q_{122} & Q_{123} & Q_{131} & Q_{132} & Q_{133} \\
Q_{211} & Q_{212} & Q_{213} & Q_{221} & Q_{222} & Q_{223} & Q_{231} & Q_{232} & Q_{233} \\
Q_{311} & Q_{312} & Q_{313} & Q_{321} & Q_{322} & Q_{323} & Q_{331} & Q_{332} & Q_{333}
\end{array}\right), \\
& Q^{(2)}=\left(\begin{array}{lllllllll}
Q_{111} & Q_{112} & Q_{113} & Q_{211} & Q_{212} & Q_{213} & Q_{311} & Q_{312} & Q_{313} \\
Q_{121} & Q_{122} & Q_{123} & Q_{221} & Q_{222} & Q_{323} & Q_{231} & Q_{322} & Q_{323} \\
Q_{131} & Q_{132} & Q_{133} & Q_{231} & Q_{232} & Q_{233} & Q_{331} & Q_{332} & Q_{333}
\end{array}\right), \\
& Q^{(3)}=\left(\begin{array}{lllllllll}
Q_{111} & Q_{121} & Q_{131} & Q_{211} & Q_{221} & Q_{231} & Q_{311} & Q_{321} & Q_{331} \\
Q_{112} & Q_{122} & Q_{132} & Q_{212} & Q_{222} & Q_{232} & Q_{312} & Q_{322} & Q_{332} \\
Q_{113} & Q_{123} & Q_{133} & Q_{213} & Q_{223} & Q_{233} & Q_{313} & Q_{323} & Q_{333}
\end{array}\right) .
\end{aligned}
$$

In [12, the authors discussed the local equivalence for states of three spin- $1 / 2$ particles by fixing a canonical point on a generic orbit. And they presented an explicit finite set of polynomial invariants to characterize the generic equivalent classes of three spin-1/2 particles under local unitary transformations. In this paper we discuss the nongeneric classes in terms of the canonical point. 
We use the notations in [12] by defining $X_{i i^{\prime}}=\sum_{j k} Q_{i j k} Q_{i^{\prime} j k}, Y_{j j^{\prime}}=\sum_{i k} Q_{i j k} Q_{i j^{\prime} k}$, $Z_{k k^{\prime}}=\sum_{i j} Q_{i j k} Q_{i j k^{\prime}}$. The matrices $X, Y, Z$ are hermitian and positive. So they can be diagonalized by proper rotations $L, M, N$ respectively. And one can always arrange these diagonal entries in decreasing order. In generic case, these entries are different, and the only remaining transformations which keep the $X, Y$ and $Z$ in these diagonal forms are the local unitary transformations which induce the orthogonal transformations such that $L, M$ and $N$ are one of the matrices $\operatorname{diag}(1,-1,-1) \operatorname{diag}(-1,1,-1)$, or $\operatorname{diag}(-1,-1,1)$.

On the generic orbits $X, Y$ and $Z$ have different eigenvalues respectively, and all the components of $L \alpha, M \beta, N \gamma$ are not zero when $X, Y, Z$ are transformed into diagonal matrices by some $L, M$ and $N$. A canonical point on the generic orbit is uniquely given by specifying that $X, Y$ and $Z$ are diagonalized by some $L, M$ and $N$ and have distinct eigenvalues, while all the components of $L \alpha(M \beta$ resp. $N \gamma)$ have the same sign. The generic orbits are thus parameterized by the components of $\alpha, \beta, \gamma, X, Y, Z$ and $Q$ at the canonical point on an orbit. The invariants that characterize the generic orbits are [12]

$$
\begin{aligned}
& \operatorname{tr} X^{r}, \operatorname{tr} Y^{r}, \operatorname{tr} Z^{r}, \alpha^{T} X^{r-1} \alpha, \beta^{T} Y^{r-1} \beta, \gamma^{T} Z^{r-1} \gamma \\
& \left(\alpha, X \alpha, X^{2} \alpha\right),\left(\beta, Y \beta, Y^{2} \beta\right),\left(\gamma, Z \gamma, Z^{2} \gamma\right), \\
& \alpha^{T} X^{r-1} R Y^{s-1} \beta, \alpha^{T} X^{r-1} S Z^{s-1} \gamma, \beta^{T} Y^{r-1} T Z^{s-1} \gamma, \\
& \sum_{i j k}\left(X^{r-1} \alpha\right)_{i}\left(Y^{s-1} \beta\right)_{j}\left(Z^{t-1} \gamma\right)_{k} Q_{i j k}=\alpha^{T} X^{r-1} Q^{(1)}\left(Y^{s-1} \otimes Z^{t-1}\right)(\beta \otimes \gamma),
\end{aligned}
$$

where $r, s, t=1,2,3,\left(\alpha, X \alpha, X^{2} \alpha\right)=\epsilon_{i j k} \alpha_{i}(X \alpha)_{j}\left(X^{2} \alpha\right)_{k}$. From these invariants, one can uniquely solve the parameters $\alpha, \beta, \gamma, R, S, T$ and $Q$. Hence the equivalent class of generic states are completely described by these invariants.

For the nongeneric case, the invariants above are not enough to specify an orbit under local unitary transformations. We first discuss the non generic case that there is one and only one zero component in $\alpha$ or $\beta$ or $\gamma$ at the canonical point on an orbit. Without loss of generality, we suppose $\alpha_{3}=0$ and $\alpha_{i} \beta_{j} \gamma_{k} \neq 0, i=1,2, j, k=1,2,3$. Here we may specify a canonical point by specifying that $\alpha_{1} \geq 0, \alpha_{2} \geq 0$ and the components of $\beta$ (resp. $\gamma$ ) have the same sign. From the invariants (2)-(5) one can solve $\alpha_{i}, \beta_{j}, \gamma_{k}$ and all the components of $R, S, T, Q$, except for $R_{3 i}, S_{3 i}$ and $Q_{3 i j}, i, j=1,2,3$. To solve these components, we need the following extra invariants:

$$
\left(\alpha, X \alpha, R Y^{r-1} \beta\right),\left(\alpha, X \alpha, S Z^{r-1} \gamma\right),\left(\alpha, X \alpha, Q^{(1)}\left(Y^{r-1} \otimes Z^{s-1}\right)(\beta \otimes \gamma)\right)
$$

where $r, s=1,2,3$. In the case $\alpha_{3}=0$, there are three invariants from the first and the 
second expressions of (6) respectively:

$$
\alpha_{1} \alpha_{2}\left(x_{2}^{2}-x_{1}^{2}\right) \sum R_{3 i} y_{i}^{2(r-1)} \beta_{i}, \quad \alpha_{1} \alpha_{2}\left(x_{2}^{2}-x_{1}^{2}\right) \sum S_{3 i} z_{i}^{2(r-1)} \gamma_{i},
$$

and nine invariants from the last formula:

$$
\alpha_{1} \alpha_{2}\left(x_{2}^{2}-x_{1}^{2}\right) \sum Q_{3 i j} y_{i}^{2(r-1)} z_{j}^{2(s-1)} \beta_{i} \gamma_{j}
$$

They can be written in matrix forms:

$$
\alpha_{1} \alpha_{2}\left(x_{2}^{2}-x_{1}^{2}\right) \Lambda F R_{3}, \quad \alpha_{1} \alpha_{2}\left(x_{2}^{2}-x_{1}^{2}\right) \Theta G S_{3}, \quad \alpha_{1} \alpha_{2}\left(x_{2}^{2}-x_{1}^{2}\right)(\Lambda F) \otimes(\Theta G) Q_{3},
$$

where:

$$
\Lambda=\left(\begin{array}{ccc}
1 & 1 & 1 \\
y_{1}^{2} & y_{2}^{2} & y_{3}^{2} \\
y_{1}^{4} & y_{2}^{4} & y_{3}^{4}
\end{array}\right), \Theta=\left(\begin{array}{ccc}
1 & 1 & 1 \\
z_{1}^{2} & z_{2}^{2} & z_{3}^{2} \\
z_{1}^{4} & z_{2}^{4} & z_{3}^{4}
\end{array}\right)
$$

$F=\operatorname{diag}\left(\beta_{1}, \beta_{2}, \beta_{3}\right), G=\operatorname{diag}\left(\gamma_{1}, \gamma_{2}, \gamma_{3}\right), R_{3}=\left(R_{31}, R_{32}, R_{33}\right)^{T}, S_{3}=\left(S_{31}, S_{32}, S_{33}\right)^{T}$, $Q_{3}=\left(Q_{311}, Q_{312}, Q_{313}, Q_{321}, Q_{322}, Q_{323}, Q_{331}, Q_{332}, Q_{333}\right)^{T}$. Since we assume that $\operatorname{det}(\Lambda F)$ and $\operatorname{det}(\Theta G)$ are not zero, the components $R_{3 i}, S_{3 i}$ and $Q_{3 i j}$ can be solved from these new invariants.

When the zero entry is $\alpha_{1}$ or $\alpha_{2}$, the corresponding components $R_{1 i}, S_{1 i}, Q_{1 i j}$ or $R_{2 i}, S_{2 i}, Q_{2 i j}$ can be also computed respectively by using the invariants in (6).

Similarly, when the zero entry is in $\beta$ or $\gamma$, the extra invariants we need are

$$
\left(\beta, Y \beta, R^{T} X^{r-1} \alpha\right),\left(\beta, Y \beta, T Z^{r-1} \gamma\right),\left(\beta, Y \beta, Q^{(2)}\left(X^{r-1} \otimes Z^{s-1}\right)(\alpha \otimes \gamma)\right)
$$

or

$$
\left(\gamma, Z \gamma, S^{T} X^{r-1} \alpha\right),\left(\gamma, Z \gamma, T^{T} Y^{r-1} \beta\right),\left(\gamma, Z \gamma, Q^{(3)}\left(X^{r-1} \otimes Y^{s-1}\right)(\alpha \otimes \beta)\right)
$$

respectively, where $r, s=1,2,3$.

As an examples of the above non generic states we set $R_{i j}=S_{i j}=T_{i j}=0, \alpha_{1}=\alpha_{2}=$ $\beta_{1}=\beta_{2}=\gamma_{1}=\gamma_{2}=Q_{111}, \gamma_{3}=\beta_{3}=Q_{333}, Q_{i j k}=0$ except for $i=j=k=1,2,3$ and $Q_{111} \neq Q_{222} \neq Q_{333}$. According to the relations [13] between entries of $\rho$ and the real parameters related to $\rho$, we have the density matrix with real parameters:

$$
\rho(a, b, c)=\frac{1}{8}\left(\begin{array}{cccccccc}
1+3 c & a x & a x & 0 & a x & 0 & 0 & a+b i \\
a \bar{x} & 1-c & 0 & a x & 0 & a x & a-b i & 0 \\
a \bar{x} & 0 & 1-c & a x & 0 & a-b i & a x & 0 \\
0 & a \bar{x} & a \bar{x} & 1-c & a+b i & 0 & 0 & a x \\
a \bar{x} & 0 & 0 & a-b i & 1+c & a x & a x & 0 \\
0 & a \bar{x} & a+b i & 0 & a \bar{x} & 1+c & 0 & a x \\
0 & a+b i & a \bar{x} & 0 & a \bar{x} & 0 & 1+c & a x \\
a-b i & 0 & 0 & a \bar{x} & 0 & a \bar{x} & a \bar{x} & 1-3 c
\end{array}\right),
$$


where $a=Q_{111}, b=Q_{222}, c=Q_{333}, x=1-i, \bar{x}=1+i$. When $a= \pm 0.1, b=0, c \in$ $(-0.3,0.3)$, the matrix $\rho(a, b, c)$ is positive. Moreover for the same $c$, the invariants given above have the same values for $\rho(0.1,0, c)$ and $\rho(-0.1,0, c)$. So $\rho(0.1,0, c)$ and $\rho(-0.1,0, c)$ are equivalent under local unitary transformations, i.e. they belong to the same orbit.

When there are two zero entries in the components (on the canonical point) of $\alpha, \beta, \gamma$, the problem is much more complicate. We first consider the case that the two zero entries are in different vectors. We suppose that $\alpha_{3}=\beta_{3}=0$ and specify the canonical point in the orbit by $\alpha_{i} \geq 0, \beta_{i} \geq 0, i=1,2$. Then the invariants given above can determine all the components of $R, S, T, Q$ except for $R_{33}$ and $Q_{33 i}, i=1,2,3$. By introducing new invariants $\operatorname{tr} R R^{T}$ and $\operatorname{tr} Q^{(3) T} Z^{r-1} Q^{(3)}$ we can solve $R_{33}^{2}$ and $Q_{33 i}^{2}$ in terms of these invariants. And the new invariants $\left(Q^{(3) T} Z^{r-1} \gamma\right)^{2}, r=1,2,3$ can determine the values of $Q_{33 i} Q_{33 j}, i \neq j$. Therefore in this case we can get the value of $\left|R_{33}\right|$ and can determine $Q_{33 i}$ up to a same sign from the invariants.

For the case that the two zeros are in a same vector, we suppose $\alpha_{2}=\alpha_{3}=0$, then we can specify the canonical point as $\alpha_{1} \geq 0$ and $\beta_{i}$ (resp. $\gamma_{i}$ ) have same sign. In this case the invariants given by (2)-(8) can only give us the values of $R_{1 i}, S_{1 i}, T_{i j}$ and $Q_{1 i j}$, where $i, j=$ 1, 2, 3. The invariants $\operatorname{tr} R Y^{r-1} R^{T} X^{s-1}, \operatorname{tr} S Z^{r-1} S^{T} X^{s-1}$, and $\operatorname{tr} Q^{(1) T} Q^{(1)}\left(Y^{s-1} \otimes Z^{t-1}\right)$ can determine the values of $R_{2 i}^{2}, \quad R_{3 i}^{2}, S_{2 i}^{2}, \quad S_{3 i}^{2}$ and $Q_{k i j}^{2}, k=2,3, i, j=1,2,3$. By using the invariants $\left(X^{r-1} R Y^{s-1} \beta\right)^{2},\left(X^{r-1} S Z^{s-1} \gamma\right)^{2}$ and $\left(\left(Y^{r-1} \otimes Z^{s-1}\right) Q^{(1) T} X^{t-1} \alpha\right)^{2}$ we can further fix the values of $R_{k i} R_{k j}, S_{k i} S_{k j}$ and $Q_{k i l} Q_{k j l}, Q_{k l i} Q_{k l j}$, where $k=2,3, i, j, l=1,2,3, i \neq j$. So we can determine the values of the remaining components up to some sign.

When the zero entries are located in other places of the components of $\alpha, \beta$ and $\gamma$, the new invariants can be similarly obtained. We summarize these invariants as follows:

$$
\begin{aligned}
& \operatorname{tr} R Y^{r-1} R^{T} X^{s-1}, \operatorname{tr} S Z^{r-1} S^{T} X^{s-1}, \operatorname{tr} T Z^{r-1} T^{T} Y^{s-1}, \\
& \operatorname{tr} Q^{(1) T} X^{r-1} Q^{(1)}\left(Y^{s-1} \otimes Z^{t-1}\right), \\
& \left(X^{r-1} R Y^{s-1} \beta\right)^{2},\left(Y^{r-1} R^{T} X^{s-1} \alpha\right)^{2},\left(X^{r-1} S Z^{-1} \gamma\right)^{2}, \\
& \left(Z^{r-1} S^{T} X^{s-1} \alpha\right)^{2},\left(Y^{r-1} T Z^{s-1} \gamma\right)^{2},\left(Z^{r-1} T^{T} Y^{s-1} \beta\right)^{2}, \\
& \left(\left(Y^{r-1} \otimes Z^{s-1}\right) Q^{(1) T} X^{t-1} \alpha\right)^{2},\left(\left(X^{r-1} \otimes Z^{s-1}\right) Q^{(2) T} Y^{t-1} \beta\right)^{2}, \\
& \left(\left(X^{r-1} \otimes Y^{s-1}\right) Q^{(3) T} Z^{t-1} \gamma\right)^{2} .
\end{aligned}
$$

The sign of the parameters can be further fixed for some detailed cases. For example, with respect to the case that the two zeros are in different vectors, if not all of the invariants of $\left(\beta, Y \beta, T Z^{r-1} \gamma\right)$ or $\left(\alpha, X \alpha, S Z^{r-1} \gamma\right)$ are zero, then the entries of $T_{3 i}$ or $S_{3 i}$ are not all 
zero. We can then use invariants $\left(\alpha, X \alpha, R T Z^{r-1} \gamma\right)$ or $\left(\beta, Y \beta, R^{T} S Z^{r-1} \gamma\right)$ to determine the sign of $R_{33}$. And use the invariants $\left(\alpha, X \alpha, Q^{(1)}\left(Y^{r-1} \otimes Z^{s-1}\right) \tilde{T}\right)$ or $\left(\beta, Y \beta, Q^{(2)}\left(X^{r-1} \otimes\right.\right.$ $\left.\left.Z^{s-1}\right) \tilde{S}\right)$ to determine the sign of $Q_{33 i}$. Where $\tilde{T}=\left(T_{11}, T_{12}, T_{13}, T_{21}, T_{22}, T_{23}, T_{31}, T_{32}, T_{33}\right)^{T}$ and similarly for $\tilde{S}$.

We have investigated the invariants for non-generic three-qubit states, and presented a complete set of invariants for a class of nongeneric three-qubit quantum mixed states. Any two of these density matrices are locally equivalent if and only if all these invariants have equal values for these density matrices. For more complicated cases, e.g. there are more zero entries in the components of $\alpha, \beta, \gamma$ in the canonical point of an orbit, by adding some new invariants we can also determine the parameters $R, S, T, Q$ up to a sign. However we only know how to determine the sign by adding more new invariants for some special cases. The problem for general cases is still open.

\section{References}

[1] M.A. Nielsen and I.L. Chuang, Quantum Computation and Quantum Information, Cambridge University Press, Cambridge, 2000.

[2] K. Chen, S. Albeverio and S.M. Fei, Phys. Rev. Lett. 95, 040504 (2005); Phys. Rev. A 68, 062313 (2003).

[3] M. Horodecki, P. Horodecki and R. Horodecki, Phys. Lett. A 200, 340 (1995).

[4] P. Horodecki and R. Horodecki, Phys. Lett. A 210, 227 (1996).

[5] M. Horodecki, P. Horodecki and R. Horodecki, Phys. Lett. A 222, 21 (1996).

[6] E.M. Rains, IEEE Transactions on Information Theory 46 54-59(2000).

[7] M. Grassl, M. Rötteler and T. Beth, Phys. Rev. A 58, 1833 (1998).

[8] Y. Makhlin, Quan. Inf. Pro. 1, 243 (2002).

[9] S. Albeverio, S.M. Fei, P. Parashar, W.L. Yang, Phys. Rev. A 68, 010303 (2003).

[10] S. Albeverio, S.M. Fei and D. Goswami, Phys. Lett. A 340 37-42(2005).

[11] S. Albeverio, L. Cattaneo, S.M. Fei and X.H. Wang, Int. J. Quant. Inform. 3 (2005) 603-609; Rep. Math. Phys. 56 (2005)341-350. 
[12] N. Linden, S. Popescu, and A. Sudbery, Phy. Rev. Lett. 83, 243 (1999).

[13] T.F. Havel, Quantum Information Processing 1:511-38 (2003). 